\section{Outubro Rosa e mamografias: quando a comunicação em saúde erra o alvo}

\section{Pink October and mammograms: when health communication misses the target}

\author{
Octubre Rosa y mamografías: cuando la \\ comunicación en salud confunde el objetivo
}

\author{
Oswaldo Santos Baquero 1,2 \\ Elizabeth Angélica Salinas Rebolledo 3 \\ Adeylson Guimarães Ribeiro 4 \\ Patricia Marques Moralejo Bermudi 3 \\ Alessandra Cristina Guedes Pellini 5,6 \\ Marcelo Antunes Failla 7 \\ Breno Souza de Aguiar 3,5 \\ Carmen Simone Grilo Diniz 3 \\ Francisco Chiaravalloti Neto 3
}

doi: 10.1590/0102-311X00149620

\section{Resumo}

O câncer de mama é o tipo de câncer mais diagnosticado e a principal causa de morte por câncer na população feminina. As mamografias de rastreamento e o tratamento precoce são geralmente os meios mais utilizados na tentativa de reduzir essa mortalidade e são incentivados no Outubro Rosa, uma campanha de divulgação anual. Contudo, estudos recentes têm relacionado o aumento do rastreamento com uma maior morbimortalidade em razão do sobrediagnóstico e do sobretratamento. No presente estudo, avaliaram-se as buscas relativas ao câncer de mama e à mamografia no Google Trends, entre 2004 e 2019, em termos da tendência, da sazonalidade e da distribuição nas Unidades Federativas brasileiras. Avaliou-se também a correlação entre a quantidade de buscas no Google Trends e a quantidade de exames de rastreamento mamográfico. As duas séries tiveram um padrão sazonal com picos em outubro, e houve excesso de exames realizados fora da faixa etária recomendada. O Outubro Rosa transmitiu informações de saúde, as popularizou e induziu comportamentos relativos a informações transmitidas; três aspectos desejáveis na comunicação e na educação em saúde. Porém, gerou um excesso de mamografias de rastreamento e não incentivou a autonomia e o consentimento livre e esclarecido. $O$ Outubro Rosa mostrou o potencial da comunicação em saúde para massas e a necessidade de que as mensagens sejam alinhadas com as melhores evidências cientificas.

Mamografia; Câncer de Mama; Meios de Comunicação

\author{
Correspondência \\ O. S. Baquero \\ Departamento de Medicina Veterinária Preventiva e Saúde \\ Animal, Faculdade de Medicina Veterinária e Zootecnia, \\ Universidade de São Paulo. \\ Av. Professor Orlando Marques de Paiva 87, São Paulo, SP \\ 05508-270, Brasil. \\ baquero@usp.br \\ 1 Faculdade de Medicina Veterinária e Zootecnia, Universidade \\ de São Paulo, São Paulo, Brasil. \\ 2 Instituto de Estudos Avançados, Universidade de São Paulo, \\ São Paulo, Brasil. \\ 3 Faculdade de Saúde Pública, Universidade de São Paulo, São \\ Paulo, Brasil. \\ 4 Instituto de Ensino e Pesquisa, Hospital de Câncer de Barretos, \\ Barretos, Brasil. \\ 5 Coordenação de Epidemiologia e Informação, Secretaria \\ Municipal de Saúde de São Paulo, São Paulo, Brasil. \\ 6 Universidade Nove de Julho, São Paulo, Brasil. \\ 7 Secretaria Municipal da Saúde de São Paulo, São Paulo, Brasil.
}




\section{Introdução}

O câncer de mama é o mais diagnosticado e a principal causa de morte por câncer na população feminina mundial 1. Nas mulheres brasileiras, segundo o Instituto Nacional de Câncer José de Alencar Gomes da Silva (INCA), é o segundo mais frequente, após o câncer de pele não melanoma 2, e a primeira causa de morte por câncer 3 . Ainda no país, entre 1980 e 2016, as taxas padronizadas de mortalidade cresceram de 9 para 12 óbitos por 100 mil mulheres, sendo que as menores, em todos os períodos, foram registradas nas regiões Norte e Nordeste, e as maiores, nas Sul e Sudeste 4.

A detecção e o tratamento precoces são geralmente os meios mais utilizados na tentativa de reduzir a mortalidade por câncer de mama 5 . Nesse sentido, no Brasil, existem políticas para a detecção precoce desse câncer 6 , baseadas majoritariamente na realização da mamografia de rastreamento, que, por muito tempo, tem sido considerada o exame de escolha entre os métodos de imagem disponíveis 7 . Contudo, estudos recentes têm relacionado o aumento desse tipo de rastreamento com eventos adversos em razão do sobrediagnóstico e do sobretratamento, levantando questionamentos quanto à efetividade do rastreamento na redução da mortalidade $8,9,10,11,12$ ou mesmo propondo novos métodos de rastreamento que poderiam ser mais promissores 13 .

As diretrizes nacionais de detecção precoce elaboradas pelo INCA, com base em extensas revisões sistemáticas das melhores evidências de efetividade e segurança 5 , recomendam a realização bienal de mamografia para o rastreamento do câncer de mama na faixa etária entre 50 e 69 anos de idade 14. De acordo com uma diretriz mais antiga do mesmo órgão, nas demais faixas etárias, a mamografia de rastreamento deveria apenas ser aplicada em mulheres de alto risco, que representam cerca de $1 \%$ da população feminina 15.

O mês da divulgação do câncer de mama, também conhecido como Outubro Rosa, é uma iniciativa idealizada pela Imperial Chemical Industries (Reino Unido) em 1985, que passou a ser financiada e promovida pela farmacêutica Zeneca em 1993 e, posteriormente, assumiu uma configuração multissetorial 16. A campanha anual tem insistido na importância do rastreamento com mamografia, exame físico clínico e autoexame das mamas, o que, para alguns, traduz-se em uma prevenção mediada pela participação da população 17 e, para outros, revela estratégias de comunicação enviesadas por propósitos comerciais, sendo a participação das usuárias limitada à obediência de instruções médicas, alheias à tomada de decisão com participação efetiva e esclarecida 16.

A primeira iniciativa da campanha Outubro Rosa no Brasil aconteceu em 2002, quando um grupo de mulheres que queria trazer o movimento para o país conseguiu o apoio privado para iluminar de rosa, durante todo um mês, o Mausoléu do Soldado Constitucionalista, conhecido como Obelisco do Ibirapuera, em São Paulo. Em outubro de 2008, diversas entidades relacionadas ao câncer de mama iluminaram de rosa monumentos e prédios em suas respectivas cidades, iniciativa que foi repetida em outubro de 2009; e, desde 2010, o INCA participa do movimento, promovendo espaços de discussão sobre o câncer de mama, divulgando e disponibilizando seus materiais informativos para profissionais e para a sociedade 18 .

Se o Outubro Rosa tiver algum efeito no comportamento da população, um dos primeiros será a busca de informações sobre o tema (câncer de mama) na Internet, o que não implica, mas pode desencadear a realização de procedimentos de rastreamento. Se esse for o caso, é possível que o tipo de informação pesquisada e/ou a realização de procedimentos de rastreamento tenham sazonalidade associada ao mês de outubro.

O mecanismo de pesquisa do Google é amplamente utilizado em todo o mundo, e podem-se acessar as tendências dos termos de pesquisa de interesse por meio da ferramenta Google Trends (https://trends.google.com). Essas tendências podem ser filtradas por data e local, de modo a se avaliar a sua associação com o Outubro Rosa. Adicionalmente, os dados relativos às mamografias de rastreamento podem ser caracterizados pela data de realização e, portanto, também permitem avaliar sua sazonalidade.

No presente estudo, avaliaram-se as buscas relativas ao câncer de mama e à mamografia no Google Trends, em termos da tendência, da sazonalidade e da distribuição nas Unidades Federativas (UF) brasileiras. Foram avaliados também a associação entre a série histórica dessas buscas e a série histórica de mamografias de rastreamento realizadas nos serviços públicos do Sistema Único de Saúde (SUS) e o seu alinhamento com as recomendações oficiais baseadas em evidências. 


\section{Métodos}

No Google Trends, o volume total de pesquisa para um termo específico em determinada data e local é dividido pelo volume total de pesquisa realizada nessa data e local. O volume de pesquisa relativo (VPR) resultante é distribuído em uma escala de 0 a 100, dividindo todos os valores pelo maior valor e multiplicando-os por 100 19. VPR baixos são excluídos. Para determinado país e período, o Google Trends retorna a série temporal de VPR para todo o país, um único VPR para as divisões geográficas escolhidas e uma lista das consultas relacionadas aos termos de pesquisa. Para períodos maiores que um ano, a série temporal resultante permanece com resolução mensal.

No Brasil, as tendências do Google estão disponíveis para UF e cidades. No presente estudo, foram escolhidas as UF, já que no nível municipal havia pouca informação disponível. O período estudado foi de 1o de janeiro de 2004 a 31 de dezembro de 2019, utilizando-se as palavras-chave: "câncer de mama" + "cancer de mama" (mesmo termo com ortografia incorreta) e "mamografia", usando todas as categorias de busca disponíveis no Google Trends. As buscas foram realizadas em 16 de março de 2020.

Para avaliar a cobertura e o alinhamento da oferta de mamografia de rastreamento às diretrizes nacionais, foram considerados apenas os procedimentos realizados no SUS. O número total de mamografias de rastreamento (código: 0204030188, Qtd. aprovada), com resolução mensal por UF de residência, de 1ํ de fevereiro de 2010 a 31 de dezembro de 2019, foi obtido do Sistema de Informações Ambulatoriais do SUS (SIA/SUS; https://datasus.saude.gov.br/acesso-a-informacao/producao-am bulatorial-sia-sus/, acessado em 02/Mar/2020).

Estimou-se a população feminina usuária do SUS como a diferença entre o total da população feminina (Instituto Brasileiro de Geografia e Estatística; https://datasus.saude.gov.br/populacaoresidente, acessado em 02/Mar/2020) e as beneficiárias de planos privados de saúde (Agência Nacional de Saúde Suplementar; http://www.ans.gov.br/anstabnet/cgi-bin/dh?dados/tabnet_br.def, acessado em 21/Jan/2020), por faixa etária, ano e UF, para o Brasil. Utilizaram-se os seguintes indicadores: (1) Razão de mamografia de rastreamento 20 :

Número de mamografia de rastreamento na faixa etária de 50 a 69 anos, em residentes de um dado local e período Metade da população feminina nessa faixa etária no respectivo local e período

A razão igual a 1 indica que a oferta de exames é suficiente para atender à população-alvo. Dada a recomendação do INCA de rastreamento bienal, o denominador corresponde à metade da população. É utilizado como proxy da cobertura, pois, nos dados disponibilizados, os procedimentos não são individualizados por mulher (pode haver mais de um exame por mulher em um mesmo ano).

(2) Razão de mamografia de rastreamento na população de alto risco:

Número de mamografia de rastreamento por faixa etária, em residentes de um dado local e período

$1 \%$ da população feminina nessa faixa etária no respectivo local e período

Aqui utilizou-se, como denominador, $1 \%$ do total da população feminina usuária do SUS, devido a uma recomendação anterior de cobertura anual de rastreamento na população de alto risco, estimada com base em $1 \%$ da população feminina com 35 anos ou mais nas populações europeias ou da América do Norte 15 , nas quais cerca de $5 \%$ dos casos da doença ocorrem em mulheres com alto risco para o desenvolvimento desse câncer 21 . Segundo o INCA, ainda não foram identificadas estratégias diferenciadas e eficazes de rastreamento para a redução da mortalidade nesse subgrupo; assim, o órgão recomenda o seguimento clínico individualizado para essas mulheres.

(3) Razão de mamografia de rastreamento nas faixas etárias contrárias às recomendações:

Número de mamografia de rastreamento por faixa etária contrária às recomendações, em residentes de um dado local e período

Metade da população feminina nessa faixa etária no respectivo local e período 
Para fins de comparação com o primeiro indicador, calculou-se a razão anual de exames de mamografia de rastreamento nas faixas etárias contrárias às recomendações: 35-49 anos, 70-74 anos e 75 anos ou mais, considerando a metade da população nessas faixas etárias. Também foi calculada a média do período.

Foi calculada ainda a diferença do número de mamografias bilaterais para rastreamento realizadas no Brasil em relação ao número esperado para a população-alvo estimada por faixa etária (metade da população entre os 50-69 anos de idade e 1\% da população com 35 anos ou mais pertencente à população de alto risco).

As análises da tendência e da sazonalidade das séries temporais e da associação entre as buscas no Google Trends e as mamografias de rastreamento nas UF foram descritivas, sendo esta última complementada com o coeficiente de correlação de Pearson (nível de significância estatística de 5\%). Para correção das diferenças etárias entre as UF, as razões de mamografia foram padronizadas por idade, utilizando-se a população mundial padrão da Organização Mundial da Saúde (OMS).

\section{Resultados}

A tendência de buscas no Google por câncer de mama diminuiu entre 2005 e 2009. Houve aumento no padrão sazonal com picos em outubro, desde 2010, para câncer de mama, e desde 2013, para mamografia. A partir de 2014, os picos se mostraram marcadamente altos (Figura 1a). As mamografias de rastreamento aumentaram entre 2010 e 2013 e, desde então, apresentaram um padrão sazonal com picos em outubro e leve tendência de queda a partir de 2014 (Figura 1b).

Em relação ao número de mamografias de rastreamento por faixa etária, observou-se elevado número de exames realizados fora da faixa recomendada. A média anual da razão de mamografia de rastreamento por grupos de idade (indicadores 1 e 3) foi de 0,18 entre 35 e 49 anos; 0,34 entre 50 e

\section{Figura 1}

Volume de pesquisa relativo (VPR) no Google Trends para câncer de mama e mamografia entre 2004 e 2019 e número de mamografias de rastreamento no Sistema de Informações Ambulatoriais do Sistema Único de Saúde (SIA/SUS). Brasil, 2010 a 2019.

1a) Google Trends

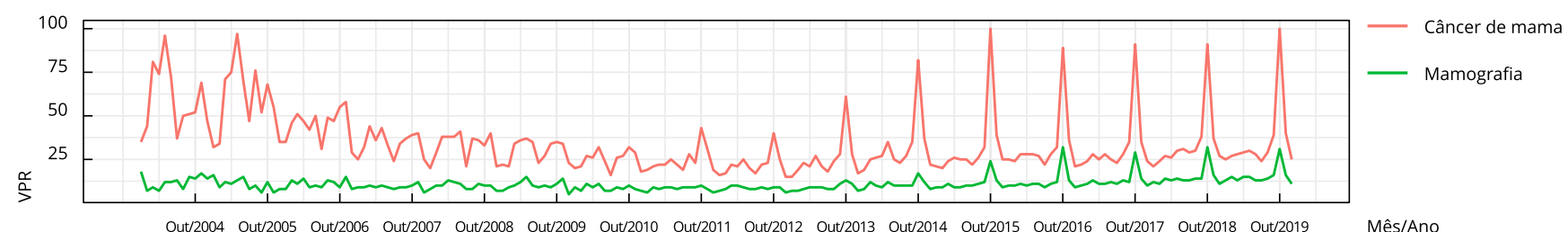

1b) Mamografias de rastreamento

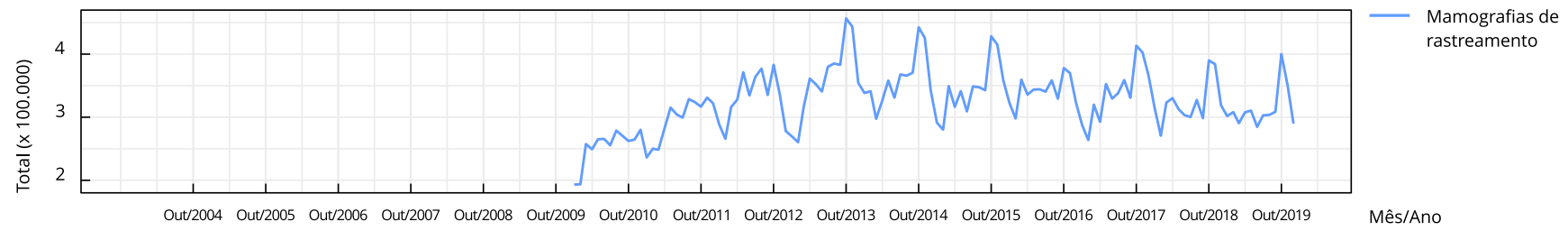

Fonte: Google Trends (https://trends.google.com, acessado em 16/Mar/2020) e SIA/SUS (https://datasus.saude.gov.br/acesso-a-informacao/producaoambulatorial-sia-sus/, acessado em 02/Mar/2020). 
69; 0,17 entre 70 e 74; e de 0,07 em mulheres de 75 anos ou mais. Durante todo o período, no grupo etário entre 35 e 49 anos, foram realizadas 13.879 .836 mamografias de rastreamento, correspondentes a $35,5 \%$ do total; $22.925 .815(58,6 \%)$ em conformidade com as recomendações, ou seja, entre 50 e 69 anos. Ainda, foram realizadas 2.294.771 mamografias de rastreamento em mulheres com 70 anos ou mais, correspondentes a 5,9\% do total.

Entre 2010 e 2014, houve uma tendência crescente no número de mamografias de rastreamento nas mulheres de 50 a 69 anos de idade, que depois estabilizou-se. No grupo de 35 a 49 anos, também se observou uma tendência crescente de rastreamento; porém, mais discreta entre 2010 e 2013 que, após esse período, tornou-se decrescente (Figura 2).

A quantidade mensal de mamografias de rastreamento em todas as faixas etárias apresentou sazonalidade com picos em outubro, principalmente a partir de 2013, mas a sazonalidade não foi tão evidente na faixa etária de 35 a 49 anos (Figura 2).

Como visto, na faixa etária recomendada para o rastreamento (50 a 69 anos), a média anual da razão de mamografias de rastreamento realizadas, em relação à necessidade estimada desse exame nessa faixa etária (indicador 1), foi inferior a 0,35; foram realizados 35 exames a cada 100 mulheres da população-alvo, e, ainda, tem diminuído levemente nos últimos cinco anos. Porém, na razão de mamografia de rastreamento estimada para cobrir a população de alto risco (indicador 2), os valores ultrapassaram, de longe, o esperado se as mamografias fossem realizadas apenas na população de alto risco, estimada em 1\% da população feminina total, sobretudo entre 35 e 49 anos e entre 70 e 74 anos de idade, ainda que essa tendência também venha diminuindo nos últimos cinco anos (Figura 3).

A diferença do número de mamografias bilaterais para rastreamento realizadas no Brasil em relação ao número esperado por faixa etária mostra que, em média, houve um déficit anual de 4,5 milhões de mamografias de rastreamento na faixa etária preconizada (50 a 69 anos) e um excedente nas demais faixas etárias, considerando a população de alto risco, que superou 1 milhão (1,42 milhão na média anual).

\section{Figura 2}

Total de mamografias para rastreamento realizadas mensalmente na população de mulheres usuárias do Sistema Único de Saúde (SUS), segundo faixa etária. Brasil, 2010 a 2019.

2a) Maiores de 70 anos

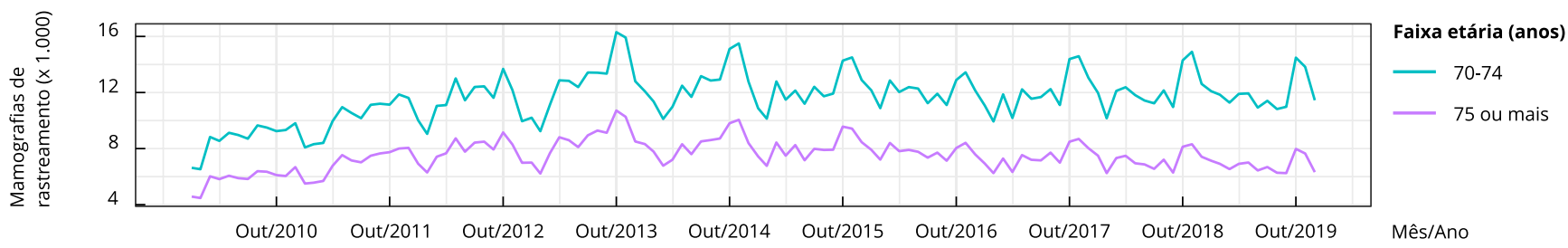

2b) Menores de 70 anos

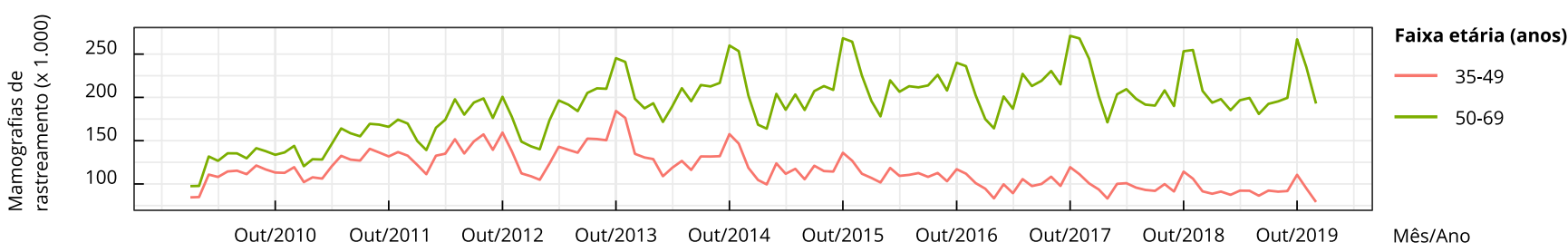

Fonte: Sistema de Informações Ambulatoriais do SUS (SIA/SUS; https://datasus.saude.gov.br/acesso-a-informacao/producao-ambulatorial-sia-sus/, acessado em 02/Mar/2020). 
Figura 3

Razão de mamografias de rastreamento realizadas na população de mulheres usuárias do Sistema Único de Saúde (SUS) para a população-alvo (indicador 1) e para a população de alto risco por faixa etária contrária às recomendações (indicador 2). Brasil, 2010 a 2019.

3a) 50-69 anos

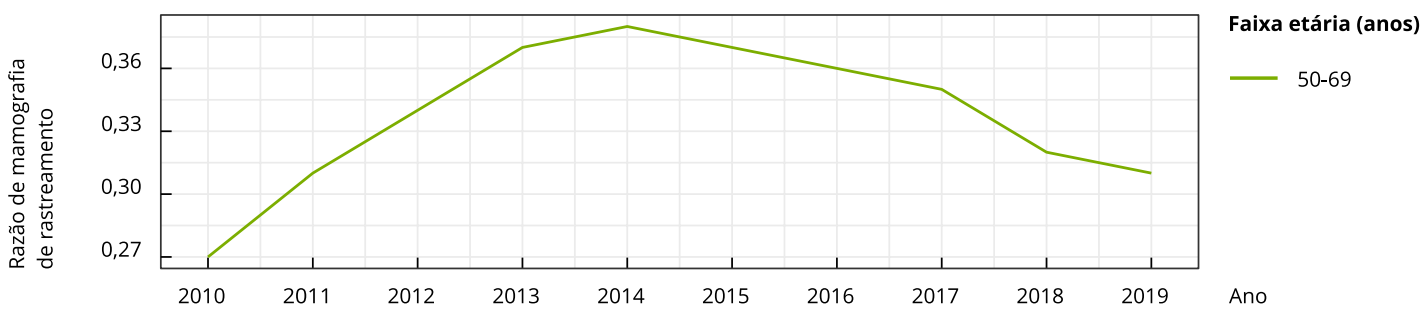

3b) Faixas etárias contrárias às recomendações

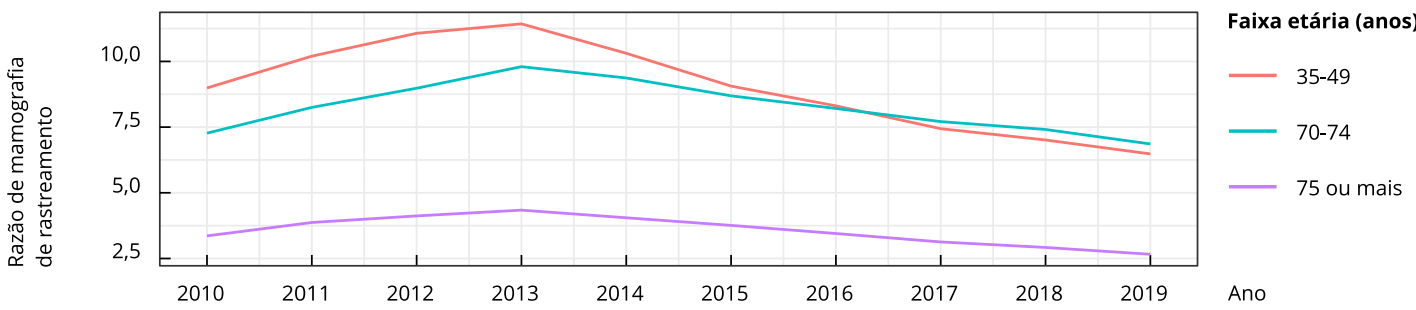

Fonte: Google Trends (https://trends.google.com, acessado em 16/Mar/2020) e SIA/SUS (https://datasus.saude.gov.br/acesso-a-informacao/producaoambulatorial-sia-sus/, acessado em 02/Mar/2020).

Em relação às pesquisas no Google, o VPR de câncer de mama variou de 66 a 100 entre as UF brasileiras, e o VPR de mamografia variou de 61 a 100 (Figura 4). Essas distribuições dos VPR não consideraram as UF de Roraima e Distrito Federal que, para câncer de mama e mamografia, respectivamente, tiveram VPR menores do que o VPR mínimo retornado pelo Google Trends.

Os VPR de câncer de mama e mamografia tiveram uma distribuição espacial semelhante (Figura 4); os coeficientes de variação foram iguais a 0,12 e 0,14 , respectivamente, enquanto o coeficiente de correlação entre ambas as variáveis foi igual a 0,98 (IC95\%: 0,96-0,99; p <0,001).

As maiores razões de mamografia de rastreamento ocorreram nas UF das regiões Sudeste e Sul (Figura 5). Não houve correlação estatisticamente significativa entre o VPR de mamografia e as razões de mamografia de rastreamento nas diferentes faixas etárias.

\section{Discussão}

As séries históricas da procura por câncer de mama e mamografia no Google, bem como as mamografias de rastreamento, apresentaram sazonalidade e picos em outubro. Esse padrão foi relatado em estudos prévios baseados no Google Trends 22,23,24, mostrando (sob o pressuposto de que não houve fatores desconhecidos e capazes de gerar picos da magnitude observada em outubro) a efetividade do Outubro Rosa para alterar o interesse pelo câncer de mama e o curto prazo do efeito.

O efeito do Outubro Rosa é diferenciado quando comparado a outras campanhas. Nos Estados Unidos e no Brasil, o volume de procura e o tamanho dos picos no respectivo mês de conscientização foram maiores quando comparados ao câncer de próstata 22,24. A maior visibilidade do câncer de 


\section{Figura 4}

Volume de pesquisa relativo (VPR) no Google Trends para câncer de mama e mamografia nas Unidades Federadas brasileiras, 2004 a 2019.

4a) Câncer de mama

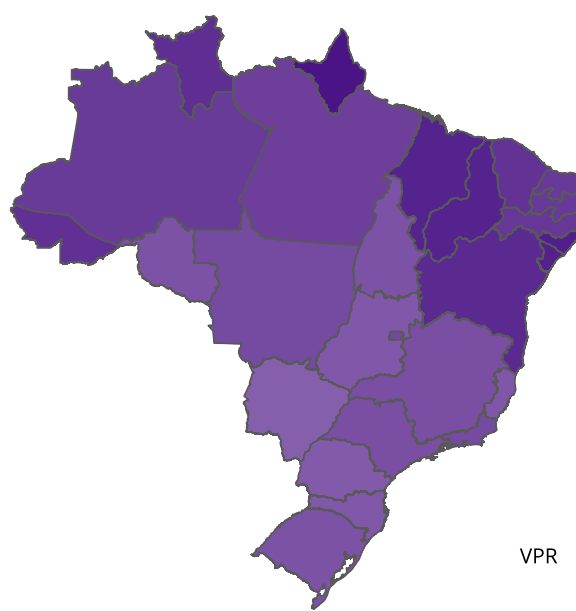

4b) Mamografia

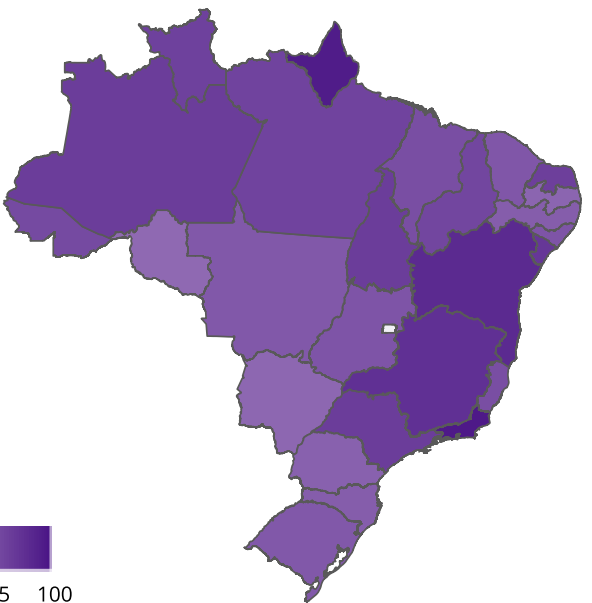

Fonte: Google Trends (https://trends.google.com, acessado em 16/Mar/2020).

mama pode explicar parte dessa efetividade diferencial, mas a existência de outras campanhas sobre esse câncer que não se concentram em outubro 2,5,25 e não se manifestam na série histórica com a intensidade dos picos em outubro indica que a efetividade diferencial não se deve simplesmente ao tipo de câncer. De qualquer forma, a efetividade demonstrada por esses estudos refere-se à capacidade de induzir à procura de informações sobre o câncer de mama na Internet, algo potencialmente relacionado, mas não necessariamente capaz de estimular comportamentos associados à detecção precoce ou de reduzir as taxas de morbidade e mortalidade pela doença.

No Brasil, o Outubro Rosa, além de induzir à busca por câncer de mama em si, levou também à busca por mamografia no Google 23. Nos Estados Unidos, entre 1994 e 2003, houve sazonalidade do número de mamografias de rastreamento com picos em outubro, mas somente durante os quatro primeiros anos 26. Esses picos de mamografias realizadas nos Estados Unidos foram menores que os picos de busca por mamografia no Google no Brasil, do que se pode concluir que o efeito do Outubro Rosa não é tão acentuado na realização de mamografias nos Estados Unidos. No entanto, vale considerar que se trata de duas evidências de informações, lugares e momentos diferentes, e os dados não descartam que, entre 1998 e 2003, o Outubro Rosa tenha incentivado a realização de mamografias nos Estados Unidos, ainda que não de forma sazonal.

No presente estudo, encontrou-se uma sazonalidade, com picos em outubro, tanto na busca por mamografia no Google como na realização de mamografia de rastreamento. As buscas por câncer de mama no Google tiveram uma tendência decrescente entre 2004 e 2009. No fim desse período e nos anos seguintes, ocorreu o acolhimento institucional do Outubro Rosa, inclusive pelo próprio INCA, e, concomitantemente, houve uma tendência crescente no número de mamografias de rastreamento. A sazonalidade nas buscas do Google apareceu após esse acolhimento e foi posterior à tendência crescente mencionada. Isso é compatível com a hipótese de que os efeitos da campanha não foram mediados exclusivamente pela Internet, e que a institucionalização intersetorial da campanha, com o aumento da oferta dos exames, foi decisiva para consolidar o regime de rastreamento por vários meios.

As buscas no Google por câncer de mama e mamografia tiveram uma correlação elevada e foram relativamente homogêneas nas diferentes UF. No entanto, a realização de mamografia de rastrea- 
Figura 5

Razão de mamografias de rastreamento na população de mulheres usuárias do Sistema Único de Saúde (SUS), padronizada por faixa etária, segundo a população-alvo (indicador 1) e segundo a população de alto risco por faixa etária contrária às recomendações (indicador 2), para as Unidades Federadas brasileiras, 2010 a 2019.

5a) $35-49$ anos

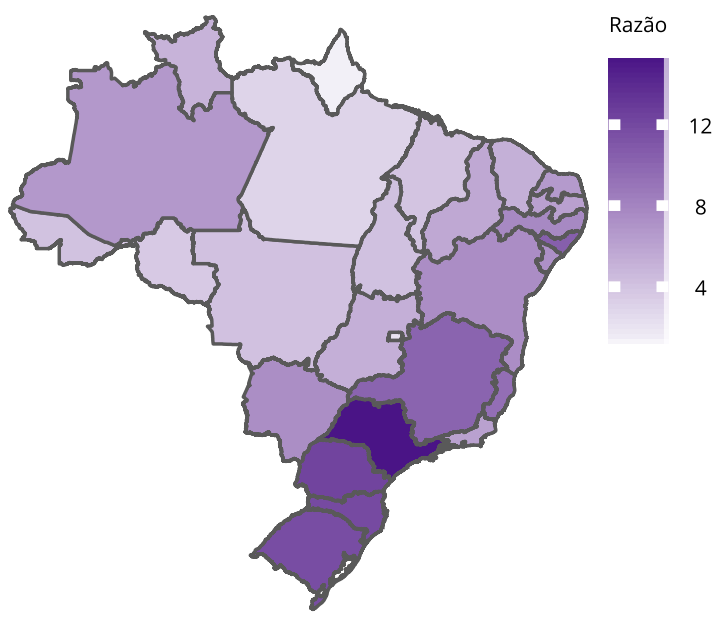

5c) $70-74$ anos

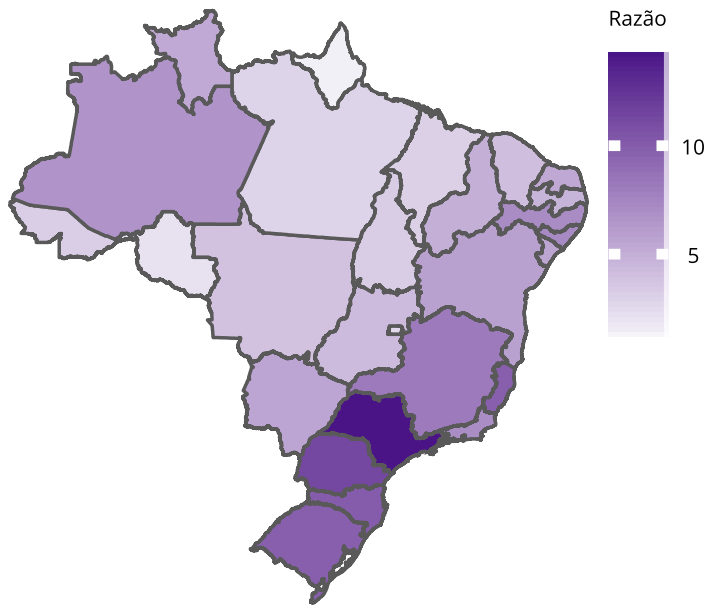

5b) 50-69 anos

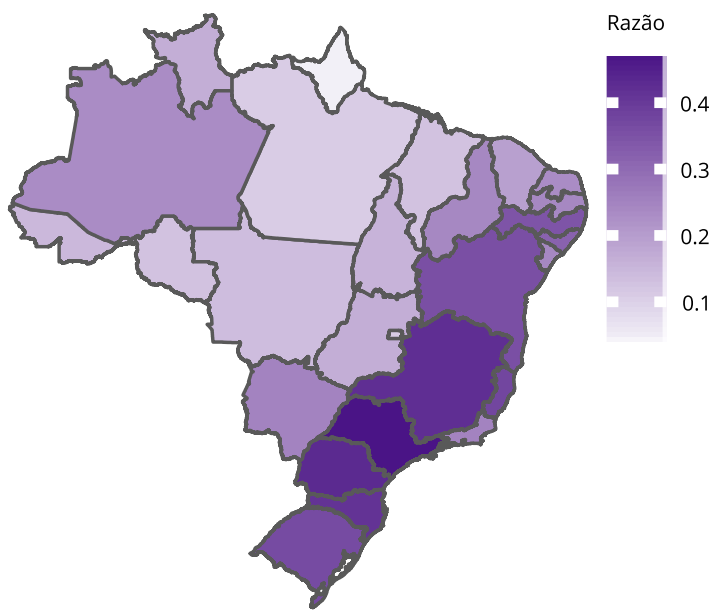

5d) 75 anos ou mais

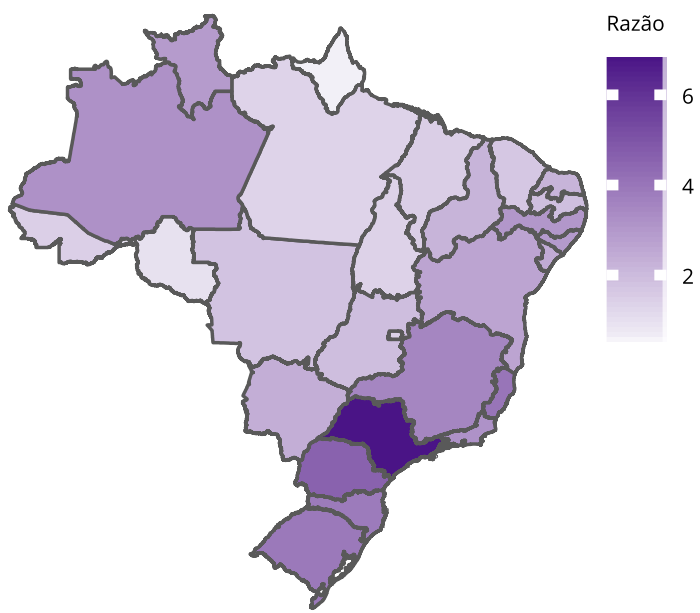

Fonte: Sistema de Informações Ambulatoriais do SUS (SIA/SUS; https://datasus.saude.gov.br/acesso-a-informacao/producao-ambulatorial-sia-sus/, acessado em 02/Mar/2020).

mento teve distribuição espacial mais variável, provavelmente devido à heterogeneidade no acesso a serviços de saúde com disponibilidade de mamografia 27 . Assim, é possível que, em alguns lugares, seja maior a proporção de mulheres sentindo necessidade e com impossibilidade de realizar mamografias para se prevenir dos efeitos do câncer de mama. Em outras palavras, o Outubro Rosa e as desigualdades no acesso a serviços de saúde podem criar uma maior carga emocional nas populações mais vulneráveis. No entanto, vale lembrar que, de acordo com alguns estudos, a realização de mamografia de rastreamento não se traduz de forma consistente em menores taxas de mortalidade 8,9 e tende a ser 
menos comum entre mulheres em situação socioeconômica vulnerável 28,29. Além disso, há evidências de associação positiva entre a taxa de mortalidade por câncer de mama e o nível socioeconômico 9,30,31. Assim, o Outubro Rosa pode ter efeitos desfavoráveis não apenas mediados pela realização de mamografias e seus desdobramentos 11,12,32, mas também pela carga emocional gerada a partir da percepção da necessidade aliada à incapacidade de realizar as mamografias.

É possível dimensionar o excesso de rastreamento fora da faixa etária recomendada, levando-se em conta que somente $1 \%$ da população feminina é considerada de alto risco para o desenvolvimento do câncer de mama 5,14. Dessa forma, nas faixas etárias para as quais existe recomendação do INCA contrária ao rastreamento, a população-alvo corresponderia apenas ao grupo de alto risco; contudo, as razões de exames de mamografia de rastreamento na população feminina usuária do SUS variaram, entre 2010 e 2019, de 2,66 a 11,43, a depender do grupo etário, sendo maior nas mulheres mais jovens (de 35 a 49 anos).

Além disso, do total de exames realizados entre 2010 e 2019 pela população feminina usuária do SUS, 35,5\% ocorreram no grupo de 35 a 49 anos de idade; e 58,6\%, na faixa etária de 50 a 69 anos de idade. Em outro estudo, constatou-se que, em 2010 e 2011, 51,2\% das mamografias foram realizadas na faixa etária recomendada (50 a 69 anos), e 44,6\% tiveram intervalo anual 33.

Nota-se, apesar disso, uma diminuição no número de mamografias realizadas na população com menos de 50 ou mais de 69 anos de idade, possivelmente refletindo a mudança na orientação para as mulheres de alto risco quanto à periodicidade do exame nas recomendações do INCA e da Agência Internacional de Pesquisa em Câncer (IARC), a partir de 201334.

Além da participação multissetorial na popularização midiática do Outubro Rosa, deve se considerar a estrutura criada para incentivar a realização de mamografias e promover a oferta de exames mais facilitada em muitos locais, tanto em serviços privados quanto em públicos. Um estudo com dados de 2016 mostrou que, no Brasil, há um excesso de mamógrafos, principalmente no setor privado, mas também no público 27 , o que pode favorecer a realização de mamografias em situações não recomendadas. Para garantir o acesso ao exame, o Ministério da Saúde estabelece que deve haver um mamógrafo para cada 240 mil habitantes; portanto, a necessidade aproximada para o rastreamento corresponderia a 869 equipamentos, e, em 2016, o número de aparelhos em uso efetivo era de 5.310, ou seja, cerca de cinco vezes maior 27.

O excedente de mamógrafos no território brasileiro não se traduz em excesso de oferta para toda a população. No presente estudo, a razão na população usuária do SUS, na faixa etária recomendada, variou entre 0,27 e 0,38 nos anos analisados (27 a 38 exames realizados a cada 100 mulheres). Apesar das limitações na comparação, em virtude das diferenças metodológicas, os valores foram menores do que o valor levantado pelo Vigitel (Vigilância de Fatores de Risco e Proteção para Doenças Crônicas por Inquérito Telefônico), que apresentou cobertura média, no Brasil, de 76,9\% em 2019 35. Nosso resultado contrasta com os $86 \%$ de cobertura do setor privado 36 e está abaixo daquela verificada por Xavier et al. 37 , de 43,7\% para o Brasil em 2012; destacando-se, entretanto, que este último trabalho considerou a cobertura em mulheres de 40 a 69 anos e sem planos de saúde. Provavelmente, o excesso de mamógrafos se concentra em regiões geográficas e grupos socioeconômicos específicos e coexiste com o acesso deficiente a serviços de saúde em outras regiões e para diferentes grupos 38,39 .

Outro motivo para as diferenças entre as coberturas de mamografia de rastreamento encontradas no presente estudo, em relação aos estudos previamente citados 35,36,37,38, pode ter origem no tipo de procedimento tabulado. Existem dois procedimentos registrados no sistema de produção ambulatorial: mamografia bilateral para rastreamento (código: 0204030188) e mamografia (código: 0204030030), este último utilizado para o seguimento de pacientes de alto risco ou com alguma alteração previamente diagnosticada nas mamas 39 .

Assim, pode ser que alguns serviços, ou pesquisadores, estejam erroneamente contabilizando essas mamografias como se fossem mamografias para rastreamento, que devem ser usadas apenas na população assintomática, sem o diagnóstico prévio de câncer de mama; ou registrando mamografias para rastreamento como se fossem mamografias (código: 0204030030) 39. Em ambos os casos, é importante conferir as normas técnicas e a qualidade dos registros, tanto por quem registra como por aqueles que utilizam essas informações.

Destaca-se, como limitação deste estudo, que não pôde ser calculada a real cobertura do rastreamento mamográfico, primeiro pela indisponibilidade do número de procedimentos realizados no 
sistema de saúde suplementar, e segundo porque, nos dados do SIA/SUS, os procedimentos não são individualizados por mulher. O SISCAN (Sistema de Informação do Câncer) seria o mais indicado para o cálculo da cobertura do rastreamento; porém, a sua implantação ainda é parcial no Brasil 20.

De um ponto de vista institucional, observa-se um desalinhamento entre as recomendações de instituições públicas, como o INCA 5,14,21, e outras entidades profissionais 40 quanto ao rastreamento mamográfico, o que também ocorre em outros países 32,41.

Enquanto o INCA preconiza o rastreamento bienal com mamografia para mulheres de 50 a 69 anos (recomendação favorável fraca) e se posiciona contra a mamografia de rastreamento nas faixas etárias abaixo de 50 (recomendação contrária forte) e acima de 69 anos (recomendação contrária fraca entre 70 e 74 e contrária forte acima de 74 anos) 5,14, o Colégio Brasileiro de Radiologia e Diagnóstico por Imagem, a Sociedade Brasileira de Mastologia e a Federação Brasileira das Associações de Ginecologia e Obstetrícia, em conjunto, recomendam o rastreamento mamográfico anual para as mulheres entre 40 e 74 anos, preferencialmente com a técnica digital (categoria A - fortes evidências); a partir dos 75 anos, recomendam o rastreamento com mamografia, preferencialmente digital, para as mulheres com expectativa de vida maior que 7 anos, baseada nas comorbidades (categoria D - consenso de especialistas); e rastreamento anual a partir dos 30 anos de idade para mulheres com risco elevado (a depender do risco, categorias entre B, C e D, com evidências que variam de consenso de especialistas a poucas evidências científicas) 40 .

Uma revisão sistemática 42 que avaliou as evidências referentes aos danos e benefícios do rastreamento do câncer de mama evidenciou que a mamografia para mulheres na faixa etária de 50 a 69 anos resulta em um decréscimo na mortalidade específica por câncer de mama, mas não na mortalidade por todos os tipos de câncer nem por todas as causas de mortalidade. Para as mulheres mais jovens que 50 anos e mais velhas que 69 anos, as conclusões não são consistentes quanto à redução da mortalidade, não havendo um impacto do rastreamento em todas as causas de mortalidade reportadas.

Os comportamentos preventivos de saúde ajudam a reduzir o sofrimento e os custos associados às doenças 43. A promoção desses comportamentos requer uma comunicação efetiva em nível populacional, e essa efetividade exige a conscientização sobre as doenças e descrições claras dos comportamentos preventivos de saúde. Logo, os rastreamentos de câncer de mama, com seu delicado equilíbrio entre os potenciais benefícios e danos, demandam que as recomendações de periodicidade e população-alvo sejam respeitadas 5 . Nesse sentido, os consensos acerca das melhores evidências disponíveis se expressam nas atuais diretrizes nacionais do INCA 14,21, em alinhamento com as recomendações internacionais do IARC 34 , sendo a única estratégia de rastreamento recomendada a mamografia na faixa etária de 50 a 69 anos de idade, com periodicidade bienal. Ainda assim, essa deve ser considerada uma recomendação condicional, pois precisa levar em conta as preferências e os valores de cada mulher, reconhecendo a razoabilidade da decisão de não se submeter ao rastreamento, conforme declarado pelo INCA, em sua página na Internet 44: "O rastreamento com mamografia, mesmo na faixa etária recomendada, implica em riscos que precisam ser conhecidos pelas mulheres. Além dos resultados falso-positivos e falso-negativos, o rastreamento pode identificar cânceres de comportamento indolente, que não ameaçariam a vida da mulher e que acabam sendo tratados (sobrediagnóstico e sobretratamento), expondo-a a riscos e danos associados. As mulheres devem ser orientadas sobre riscos e beneficios do rastreamento mamográfico para que possam, em conjunto com o médico, decidir sobre a realização dos exames de rotina e exercer sua autonomia".

A transparência e a indicação clara de quais são e o que significam as evidências científicas contrastam com antecedentes fundamentais na popularização da mamografia de rastreamento. Klawiter (2008, apud Briatte 16) discute o papel do Breast Cancer Demonstration Project (BCDP), um projeto desenhado para promover o rastreamento mediante mamografias e exames físicos em mulheres assintomáticas que não levou em conta recomendações técnicas e evidências dos efeitos desfavoráveis do rastreamento em mulheres menores de 50 anos de idade. Além disso, o autor discute o financiamento e a influência publicitária no Outubro Rosa por parte da farmacêutica Zeneca, uma subsidiária da Imperial Chemical Industries (idealizadora do Outubro Rosa), produtora do Tamoxifen (fármaco para tratar o câncer de mama que chegou a ser o mais vendido no mundo), e uma das principais produtoras de pesticidas cujo efeito carcinógeno e a contribuição ao câncer de mama são causa de debates. $\mathrm{O}$ BCDP e a participação da Zeneca são elementos na crítica do Outubro Rosa como uma estratégia de marketing para incentivar a realização de mamografias 16 . 
No Brasil, mesmo com as recomendações do INCA, percebe-se que algumas campanhas do Outubro Rosa, sejam do setor privado 45 ou mesmo organizadas pelo SUS 46,47,48, têm estimulado o uso inapropriado da mamografia para fins de rastreamento, o que pode contribuir para a sobrecarga de um sistema de saúde já precarizado.

O Outubro Rosa transmitiu informações de saúde, as popularizou e induziu comportamentos específicos, como a busca por informações no Google e a realização de mamografias; três aspectos desejáveis na comunicação e na educação em saúde. Porém, gerou um excesso de mamografias de rastreamento e não incentivou a autonomia e o consentimento livre e esclarecido. O Outubro Rosa mostrou o potencial da comunicação em saúde para as massas e a necessidade de que as mensagens sejam alinhadas com as melhores evidências científicas.

Por fim, é necessário considerar que pode haver conflitos de interesse nas políticas de saúde pública, refletidos no desencontro entre as recomendações baseadas em evidências do Ministério da Saúde e aquelas feitas por organizações profissionais financiadas pela indústria de equipamentos e medicamentos, o que pode ser reduzido por meio de informação clara às usuárias sobre os alcances e limites das campanhas de prevenção, assim como da garantia de direito à escolha informada sobre os riscos e benefícios das intervenções.

\section{Colaboradores}

O. S. Baquero contribuiu com a concepção da pesquisa, obtenção e preparação de dados do Google Trends, análise de dados, escrita e revisão do texto. E. A. S. Rebolledo, A. G. Ribeiro, P. M. M. Bermudi, A. C. G. Pellini, M. A. Failla e B. S. Aguiar contribuíram com a obtenção e preparação de dados das mamografias de rastreamento, análise de dados, escrita e revisão do texto. C. S. G. Diniz e F. Chiaravalloti Neto contribuíram com a análise de dados, escrita e revisão do texto.

\section{Informações adicionais}

ORCID: Oswaldo Santos Baquero (0000-00032695-7946); Elizabeth Angélica Salinas Rebolledo (0000-0001-9770-7392); Adeylson Guimarães Ribeiro (0000-0001-8447-8463); Patricia Marques Moralejo Bermudi (0000-0002-5825-6389); Alessandra Cristina Guedes Pellini (0000-0002-37999415); Marcelo Antunes Failla (0000-0002-90047309); Breno Souza de Aguiar (0000-0002-6621228X); Carmen Simone Grilo Diniz (0000-00020069-2532); Francisco Chiaravalloti Neto (00000003-2686-8740).

\section{Agradecimentos}

Agradecemos à Fundação de Amparo à Pesquisa do Estado de São Paulo (FAPESP; processos 2018/22100-0 e 2017/25625-4) e ao Conselho Nacional de Desenvolvimento Científico e Tecnológico (CNPq; processo 306025/2019-1). 


\section{Referências}

1. Bray F, Ferlay J, Soerjomataram I, Siegel RL, Torre LA, Jemal A. Global cancer statistics 2018: GLOBOCAN estimates of incidence and mortality worldwide for 36 cancers in 185 countries. CA Cancer J Clin 2018; 68:394-424.

2. Instituto Nacional do Câncer José Alencar Gomes da Silva. Estimativa 2020 - incidência de câncer no Brasil. https://www.inca.gov.br/si tes/ufu.sti.inca.local/files//media/document// estimativa-2020-incidencia-de-cancer-nobrasil.pdf (acessado em 05/Fev/2020).

3. Instituto Nacional do Câncer José Alencar Gomes da Silva. Atlas on-line de mortalidade. https://mortalidade.inca.gov.br/Mortalidade Web/pages/Modelo03/consultar.xhtml\#pa nelResultado (acessado em 06/Mai/2020).

4. Instituto Nacional do Câncer José Alencar Gomes da Silva. A situação do câncer de mama no Brasil: síntese de dados dos sistemas de informação. https://www.inca.gov.br/sites/ufu. sti.inca.local/files//media/document//a_situa cao_ca_mama_brasil_2019.pdf (acessado em 03/Mar/2020).

5. Migowski A, Azevedo e Silva G, Dias MBK, Diz MDPE, Sant'Ana DR, Nadanovsky P. Guidelines for early detection of breast cancer in Brazil. II - New national recommendations, main evidence, and controversies. Cad Saúde Pública 2018; 34:e00074817.

6. Instituto Nacional do Câncer José Alencar Gomes da Silva. Diretrizes para a detecção precoce do câncer de mama no Brasil. Rio de Janeiro: Instituto Nacional do Câncer José Alencar Gomes da Silva; 2015.

7. Newman J. Quality control and artifacts in mammography. Radiol Technol 1998; 70:61-76.

8. Autier P, Boniol M. Mammography screening: a major issue in medicine. Eur J Cancer 2018; 90:34-62.

9. Diniz CSG, Pellini ACG, Ribeiro AG, Tedardi MV, De Miranda MJ, Touso MM, et al. Breast cancer mortality and associated factors in São Paulo State, Brazil: an ecological analysis. BMJ Open 2017; 7:e016395.

10. Keen JD, Jørgensen KJ. Four principles to consider before advising women on screening mammography. J Womens Health (Larchmt) 2015; 24:867-74.

11. Løberg M, Lousdal ML, Bretthauer M, Kalager M. Benefits and harms of mammography screening. Breast Cancer Res 2015; 17:63.

12. Gøtzsche PC. Mammography screening is harmful and should be abandoned. J R Soc Med 2015; 108:341-5.

13. Borges Do Nascimento F, Galdino Da Rocha Pitta M, Barreto J, Rêgo M. Análise dos principais métodos de diagnóstico de câncer de mama como propulsores no processo inovativo. Arq Med 2015; 29:153-9.

14. Facina T. Diretrizes para a detecção precoce do câncer de mama no Brasil. Rev Bras Cancerol 2016; 62:59-60.
15. Instituto Nacional do Câncer José Alencar Gomes da Silva. Parâmetros para o rastreamento do câncer de mama: recomendações para gestores estaduais e municipais. https://www. inca.gov.br/publicacoes/livros/parametrostecnicos-para-o-rastreamento-do-cancer-de -mama (acessado em 03/Mar/2020).

16. Briatte F. The biopolitics of breast cancer: changing cultures of disease and activism. Sociol Health Illn 2010; 32:818-9.

17. World Health Organization. Breast cancer awareness month in october. https://www. who.int/cancer/events/breast_cancer_month/ en/ (acessado em 23/Ago/2019).

18. Outubro Rosa. Como surgiu o Outubro Rosa. http://www.outubrorosa.org.br/historia.htm (acessado em 23/Ago/2019).

19. Google Trends. FAQ about Google Trends data. https://support.google.com/trends/ answer $/ 4365533$ ? $\mathrm{hl}=$ en\&ref_topic $=6248052$ (acessado em 06/Mai/2020).

20. Instituto Nacional do Câncer José Alencar Gomes da Silva. Ficha técnica de indicadores relativos às ações de controle do câncer de mama. Rio de Janeiro: Instituto Nacional do Câncer José Alencar Gomes da Silva; 2014.

21. Instituto Nacional do Câncer José Alencar Gomes da Silva. Câncer de mama - versão para profissionais de saúde. https://www.inca.gov. br/tipos-de-cancer/cancer-de-mama/profis sional-de-saude (acessado em 21/Jan/2020).

22. Schootman M, Toor A, Cavazos-Rehg P, Jeffe DB, McQueen A, Eberth J, et al. The utility of Google Trends data to examine interest in cancer screening. BMJ Open 2015; 5:6678.

23. Vasconcellos-Silva PR, Carvalho DBF, Trajano V, de La Rocque LR, Sawada ACMB, Juvanhol LL. Using Google Trends data to study public interest in breast cancer screening in Brazil: why not a Pink February? JMIR Public Health Surveill 2017; 3:e17.

24. Quintanilha LF, Souza LN, Sanches D, Demarco RS, Fukutani KF. The impact of cancer campaigns in Brazil: a Google Trends analysis. Ecancermedicalscience 2019; 13:963.

25. Grupo Reviver. Faça sua mamografia em agosto - calendário. http://gruporeviver.com/facasua-mamografia-em-agosto-calendario/ (acessado em 21/Jan/2020).

26. Stat bite: effect of breast cancer awareness month on mammography use. J Natl Cancer Inst 2005; 97:1493.

27. Miranda S, Patrocínio A. Distribuição de mamógrafos por macrorregião do Brasil. In: Anais do V Congresso Brasileiro de Eletromiografia e Cinesiologia e X Simpósio de Engenharia Biomédica. https://www.even3. com.br/anais/cobecseb/78881-distribuicaode-mamografos-por-macrorregiao-do-brasil/ (acessado em 03/Mar/2020). 
28. Bezerra HS, Melo TFV, Barbosa JV, Feitosa EELC, Sousa LCM. Avaliação do acesso em mamografias no Brasil e indicadores socioeconômicos: um estudo espacial. Rev Gaúcha Enferm 2018; 39:e20180014.

29. Borges ZS, Wehrmeister FC, Gomes AP, Gonçalves H. Exame clínico das mamas e mamografia: desigualdades nas regiões Sul e Nordeste do Brasil. Rev Bras Epidemiol 2016; 19:1-13.

30. Girianelli VR, Gamarra CJ, Azevedo e Silva G. Disparities in cervical and breast cancer mortality in Brazil. Rev Saúde Pública 2014; 48:459-67.

31. Bermudi PMM, Pellini AGC, Rebolledo EAS, Diniz CSG, Aguiar BS, Ribeiro AG, et al. Padrão espacial da mortalidade por câncer de mama e colo do útero na cidade de São Paulo. Rev Saúde Pública 2020; 54:142.

32. Doval MB, Volij C, Weisbrot M, Terrasa S. Controversias sobre el tamizaje de cáncer mamario con mamografía. Evid Actual Práct Ambul 2019; 22:e02023.

33. Tomazelli JG, Migowski A, Ribeiro CM, Assis M, Abreu DMF. Avaliação das ações de detecção precoce do câncer de mama no Brasil por meio de indicadores de processo: estudo descritivo com dados do Sismama, 2010-2011. Epidemiol Serv Saúde 2017; 26:61-70.

34. Lauby-Secretan B, Scoccianti C, Loomis D, Benbrahim-Tallaa L, Bouvard V, Bianchini F, et al. Breast-cancer screening: viewpoint of the IARC Working Group. N Engl J Med 2015; 372:2353-8.

35. Ministério da Saúde. Vigitel Brasil 2019: vigilância de fatores de risco e proteção para doenças crônicas por inquérito telefônico. https://www.saude.gov.br/images/pdf/2020/ April/27/vigitel-brasil-2019-vigilancia-fato res-risco.pdf (acessado em 03/Mar/2020).

36. Malta DC, Bernal RTI, Vieira Neto E, Curci KA, Pasinato MTM, Lisbôa RM, et al. Trends in risk and protective factors for non-communicable diseases in the population with health insurance in Brazil from 2008 to 2015. Rev Bras Epidemiol 2018; 21 Suppl 1:e180020.

37. Xavier DR, Oliveira RAD, Matos VP, Viacava F, Carvalho CC. Cobertura de mamografias, alocação e uso de equipamentos nas Regiões de Saúde. Saúde Debate 2016; 40:20-35.

38. Amaral P, Luz L, Cardoso F, Freitas R. Distribuição espacial de equipamentos de mamografia no Brasil. Revista Brasileira de Estudos Urbanos e Regionais 2017; 19:326-41.

39. Ministério da Saúde. Portaria no 126, de 24 de fevereiro de 2014. Altera o nome ou a descrição de procedimentos na Tabela de Procedimentos, Medicamentos, Órteses, Próteses e Materiais Especiais do Sistema Único de Saúde. Diário Oficial da União 2014; 25 fev.
40. Urban LABD, Chala LF, Bauab SP, Schaefer MB, Santos RP, Maranhão NMA, et al. Recomendações do Colégio Brasileiro de Radiologia e Diagnóstico por Imagem, da Sociedade Brasileira de Mastologia e da Federação Brasileira das Associações de Ginecologia e Obstetrícia para o rastreamento do câncer de mama. Radiol Bras 2017; 50:244-9.

41. Jin J. Breast cancer screening guidelines in the United States. JAMA 2015; 314:1658.

42. Mandrik O, Zielonke N, Meheus F, Severens JLH, Guha N, Herrero Acosta R, et al. Systematic reviews as a 'lens of evidence': determinants of benefits and harms of breast cancer screening. Int J Cancer 2019; 145:994-1006.

43. Cestari MEW, Zago MMF. A prevenção do câncer e a promoção da saúde: um desafio para o Século XXI. Rev Bras Enferm 2005; 58:218-21.

44. Instituto Nacional do Câncer José Alencar Gomes da Silva. Confira as recomendações do Ministério da Saúde para o rastreamento do câncer de mama. https://www.inca.gov.br/no ticias/confira-recomendacoes-do-ministerioda-saude-para-o-rastreamento-do-cancer-de -mama (acessado em 12/Dez/2019).

45. Sociedade Brasileira de Mastologia; Instituto Oncoclínicas. Seja a melhor pessoa para você - faça mamografia. https://www.instituto-on coclinicas.org.br/outubrorosa/?gclid $=\mathrm{Cj} 0 \mathrm{KC}$ QjwrrXtBRCKARIsAMbU6bEmemCtlY2gk 7tJr6U7V_VSccBKn6k36QEPucCfEwcKZ bv5hlFiNHEaAuJEEALw_wcB (acessado em 12/Dez/2019).

46. Prefeitura Municipal de Pedreira. Secretaria Municipal de Saúde realiza Campanha de Combate ao Câncer de Mama neste sábado, 5 de outubro. https://www.pedreira.sp.gov.br/ paginas $/ \mathrm{portal} /$ noticia?id=5730 (acessado em 12/Dez/2019).

47. Prefeitura do Município de Camaragibe. Camaragibe oferece ações do Outubro Rosa. https://www.camaragibe.pe.gov.br/camaragi be-oferece-acoes-do-outubro-rosa/ (acessado em 12/Dez/2019).

48. Prefeitura Municipal de Rio Bananal. Outubro Rosa: a Secretaria de Saúde de Rio Bananal convida todas as mulheres acima de 40 anos para participarem de uma palestra sobre a prevenção ao câncer de mama. https://riobananal. es.gov.br/Notícia/outubro-rosa-a-secretariade-saude-de-rio-bananal-convida-todas-as -mulheres-acima-de-40-anos-para-participa rem-de-uma-palestra-sobre-a-prevencao-aocancer-de-mama (acessado em 12/Dez/2019). 


\section{Abstract}

Breast cancer is the most frequently diagnosed type of cancer and is the leading cause of death from cancer in the female population. Screening mammograms and early treatment are the most frequently used means to attempt to reduce this mortality and are promoted during Pink October, an annual awareness-raising campaign. However, recent studies have correlated the increase in screening with higher morbidity and mortality, due to overdiagnosis and overtreatment. The current study assessed searches related to breast cancer and mammogram in Google Trends from 2004 to 2019 in terms of trend, seasonality, and distribution in Brazilian states. The study also assessed the correlation between the number of searches in Google Trends and the number of screening mammograms. The two series showed a seasonal pattern with peaks in October, and there was an excess in tests performed outside the recommended age bracket. Pink October transmitted and popularized health information and induced behaviors related to this information, which are three desirable aspects in health communication and education. However, the campaign also generated an excess in screening mammograms and did not encourage autonomy and free and informed consent. Pink October revealed both the potential of mass communication in health and the need for messages to be aligned with the best available scientific evidence.

Mammography; Breast Neoplasms;

Communications Media

\section{Resumen}

El cáncer de mama es el tipo de cáncer más diagnosticado y la principal causa de muerte por cáncer en la población femenina. Las mamografías de rastreo y el tratamiento precoz son generalmente los medios más utilizados en la tentativa de reducir esa mortalidad, $y$ son incentivados en el Octubre Rosa, una campaña de divulgación anual. No obstante, estudios recientes han relacionado el aumento del rastreo con una mayor morbimortalidad, debido al sobrediagnóstico y al sobretratamiento. En el presente estudio se evaluaron las búsquedas relativas al cáncer de mama, y a la mamografia en Google Trends entre 2004 y 2019, en términos de tendencia, de estacionalidad y de su distribución en las Unidades Federativas brasileñas. Se evaluó también la correlación entre la cantidad de búsquedas en Google Trends y la cantidad de exámenes de rastreo mamográfico. Las dos series tuvieron un patrón estacional con picos en octubre, y hubo un exceso de exámenes realizados fuera de la franja etaria recomendada. Octubre Rosa transmitió información de salud, la popularizó e indujo a comportamientos relacionados con la información transmitida; tres aspectos deseables en la comunicación y educación en salud. Sin embargo, generó un exceso de mamografías de rastreo y no incentivó la autonomía y el consentimiento libre e informado. Octubre Rosa mostró el potencial de la comunicación en salud para las masas y la necesidad de que los mensajes estén alineados con mejores evidencias científicas.

Mamografía; Neoplasias de la Mama; Medios de Comunicación
Recebido em 11/Jun/2020

Versão final reapresentada em 01/Mar/2021

Aprovado em 04/Mar/2021 